\title{
Évaluation de la consommation et caractérisation nutritionnelle des feuilles de Tiliacora funifera
}

\author{
MANANGA Vital(1,2,3*), ITOUA OKOUANGO Yvon Simplice(1,2), MOUKASSA Wolfgon(1), BOUKOU \\ Gabriëlla Jesnaure(1), ELENGA Michel1, 2) \\ (1)Laboratoire de Nutrition et d'Alimentation Humaine (LaNAH), Faculté des Sciences et Techniques (FST), UMNG, \\ BP : 69, Brazzaville, Congo. \\ (2)Equipe Pluridisciplinaire de Recherche en Alimentation et en nutrition (EPRAN) Faculté des Sciences et \\ Techniques (FST), UMNG. \\ (3)Physiologie Animale et nutrition, Faculté des Sciences et Techniques (Université Marien Ngouabi), BP : 69, \\ Brazzaville, Congo. \\ *Auteur correspondant e-mail : manangavital@yahoo.fr, tel : 00(242) 066743151/00(242) 057038912 \\ Original submitted in on $16^{\text {th }}$ June 2020. Published online at www.m.elewa.org/journals/ on $31^{\text {st }}$ October 2020 \\ https://doi.org/10.35759/JABs.154.6
}

\section{RESUME}

Objectif: Une étude a été réalisée sur un légume feuille utilisé comme assaisonnement (épices et condiment) par la population de Komono : les feuilles Tiliacora funifera. L'objectif général de ce travail est de réaliser une évaluation de la consommation et une caractérisation de la valeur nutritive de ce condiment et épice sauvage : les feuilles Tiliacora funifera.

Méthodologie et Résultats : Une enquête transversale de consommation des feuilles Tiliacora funifera a été menée à Komono centre sur 170 ménages. La caractérisation s'est basée par la détermination de la teneur en eau, de matière sèche, en lipide totaux, en protéines totales, en glucides totaux et en cendres totales. II ressort des enquêtes que, la feuille de Tiliacora funifera est un assaisonnement connu et consommé par tous les enquêtés (100\%). La partie consommée est la feuille (100\%), dans le but de rehausser le goût du saka-saka (100\%). Saka-saka est un plat préparé à base des feuilles de manioc. Les enquêtés s'approvisionnent beaucoup plus en forêt $(95,3 \%)$ par la cueillette $(98,2 \%)$. Les feuilles de Tiliacora funifera sont consommées uniquement dans le saka-saka sous forme cuite $(98,8 \%)$. sa fréquences de consommation est de plusieurs fois par semaine $(32,9 \%)$ avec le manioc comme aliment d'accompagnement de préférence $(84,1 \%)$. Les feuilles de Tiliacora funifera ne sont pas saisonnières, elles sont consommées toutes les saisons $(92,3 \%)$. Ces feuilles procurent le gout sucré dans le saka- saka $(96,4 \%)$. Les résultats de laboratoire donnent les caractéristiques morphologiques et nutritives suivantes: longueur des feuilles $10,14 \pm 2,25 \mathrm{~cm}$, largeur des feuilles $6,16 \pm 2,35 \mathrm{~cm}$, nombre de nervures secondaires $11 \pm 3,60$, nombre de nervures principales $1 \pm 0$, longueur de pétiole $2,14 \pm 1,20 \mathrm{~cm}$. La teneur en eau représente $50,17 \pm 6,70$ de matière fraîche ; teneur en matière sèche $49,81 \pm 6,70$ de matière fraîche ; teneur en protéines : $0,57 \pm 0,74$ de matière sèche ; teneur en lipides : $5,50 \pm 1,50$ de matière sèche ; teneur en glucides $88,53 \pm 3,76$ de matière sèche. Une teneur en cendres : $5,36 \pm 3,37$ de matière sèche. L'énergie apportée par ces feuilles est de $406,0 \mathrm{Kcal}$.

Conclusion et application des résultats: Les feuilles de Tiliacora funifera sont beaucoup utilisées par la population de la Lékoumou dans la préparation du plat de saka-saka. Elles procurent une valeur ajoutée à 
ce plat en apportant une bonne saveur (bon goût). Ces feuilles auraient une bonne qualité organoleptique et méritent d'être classées dans la liste des assaisonnements (condiment et épices). Ces feuilles seraient aussi énergétiques.

Mots clés : enquête de consommation, qualité nutritive, Komono

\begin{abstract}
Objective: A study was carried out on a leaf vegetable Tiliacora funifera used as a seasoning (spices and condiment) by the population of Komono. The general objective of this work is to carry out an evaluation of the consumption and a the nutritional value of this wild condiment and spice : the leaves Tiliacora funifera. Methodology and Results: A cross-sectional survey of consumption of Tiliacora funifera leaves was carried out in Komono center on 170 households. The characterization was based on the determination of the of water content, dry matter, total fat, total protein, total carbohydrates and total ash. Surveys show that the Tiliacora funifera leaf is a seasoning known and consumed by all Respondents (100\%). The part consumed is the leaf $(100 \%)$, in order to enhance the taste of saka-saka (100\%). Saka-saka is a dish prepared from cassava leaves. Respondents obtain the leaves more from the forest (95.3\%) and through gathering (98.2\%). The leaves of Tiliacora funifera are consumed only in saka-saka in cooked form (98.8\%). Its consumption frequency is several times a week $(32.9 \%)$ with cassava as a complementary food preferably (84.1\%). The leaves of Tiliacora funifera are not seasonal, they are eaten in all seasons $(92.3 \%)$. These leaves provide the sweet taste in saka-saka (96.4\%). The laboratory results give the following morphological and nutritional characteristics: leaf length $10.14 \pm 2.25 \mathrm{~cm}$, leaf width $6.16 \pm 2.35 \mathrm{~cm}$, number of secondary veins $11 \pm 3.60$, number of main veins $1 \pm 0$, petiole length $2.14 \pm 1.20 \mathrm{~cm}$. The water content represents $50.17 \pm 6.70$ of fresh matter; dry matter content $49.81 \pm 6.70$ fresh matter; protein content: 0.57 \pm 0.74 dry matter; lipid content: $5.50 \pm 1.50$ of dry matter; carbohydrate content $88.53 \pm 3.76$ dry matter. Ash content: $5.36 \pm 3.37$ dry matter. The energy provided by this leaf is $406.0 \mathrm{Kcal}$.

Conclusion and application of results: The leaves of Tiliacora funifera are widely used by the people of Lékoumou in the preparation of the saka-saka dish. They add value to this dish by bringing a good flavor (good taste). These leaves would have a good organoleptic quality and deserve to be classified in the list of seasonings (condiment and spices). These leaves are also energetic.
\end{abstract}

Keywords: consumption survey, nutritional quality, Komono

\section{INTRODUCTION}

Dans le monde en général, en Afrique en particulier, les condiments et épices occupent une place importante dans l'amélioration des qualités organoleptiques des régimes alimentaire des populations. Certains de ces condiments peuvent être consommés en légumes, qui rehaussent la saveur de préparations culinaires notamment des sauces ; ils sont d'origine végétale (Maistre, 1964). $\mathrm{Si}$ les épices sont des substances naturelles végétales, permettant de rehausser la saveur et l'arôme des aliments (Maistre, 1964), les condiments sont des préparations renfermant un ou plusieurs végétaux qui concourent à l'assaisonnement des aliments (Gast, 1968). Les PFNL alimentaires sont connus et utilisés par les populations des pays de l'Afrique centrale (Bikoue Carolle, Essomba Hermann et al.2007). Ils contribuent pour une bonne portion à la couverture des besoins exprimés (Mialoundama et al. 2006). En effet, il est reconnu que la valorisation des ressources agroalimentaires locales sous exploitées ou peu exploitées est l'une des voies pour améliorer l'état nutritionnel des populations (Letham, 2001). Les condiments et épices utilisés renferment outre les éléments minéraux, vitamines et facteurs nutritionnels. Sans présenter des facteurs antinutritionnels rédhibitoires (Bailey, 2003), elles sont riches en fibres alimentaires et procurent une bonne sécurité alimentaire.Au Congo, plusieurs condiments et épices sont 
ajoutés aux aliments crus ou cuits afin de relever leur saveur. C'est le cas des feuilles de Tiliacora funifera. Une plante grimpante et lianescente non cultivable, largement repartie dans les forêts d'Afrique tropicale et fait partie des PFNL d'Afrique centrale. Sa tige très rigide avec beaucoup de ramification peut atteindre plusieurs mètres de hauteur avec la présence d'entre- nœuds: les feuilles alternent et sont attachées à la tige secondaire (sous forme de branche) de plusieurs centimètres de long par l'intermédiaire du pétiole. Dans le Département de la Lékoumou à Komono plus précisément, la majorité des populations utilise des produits forestiers non ligneux parmi lesquels, les feuilles de Tiliacora funifera afin de rehausser le goût ou la saveur de leur repas. Ses feuilles présentent un goût ressemblant sucré et salé. Elles sont couramment utilisées comme assaisonnement dans la préparation des plats du saka-saka par la population de Komono à cause

\section{MATERIEL ET METHODES}

Le matériel didactique est constitué d'une fiche d'enquête dans laquelle seront mentionnées des questions ouvertes et des questions fermées basées

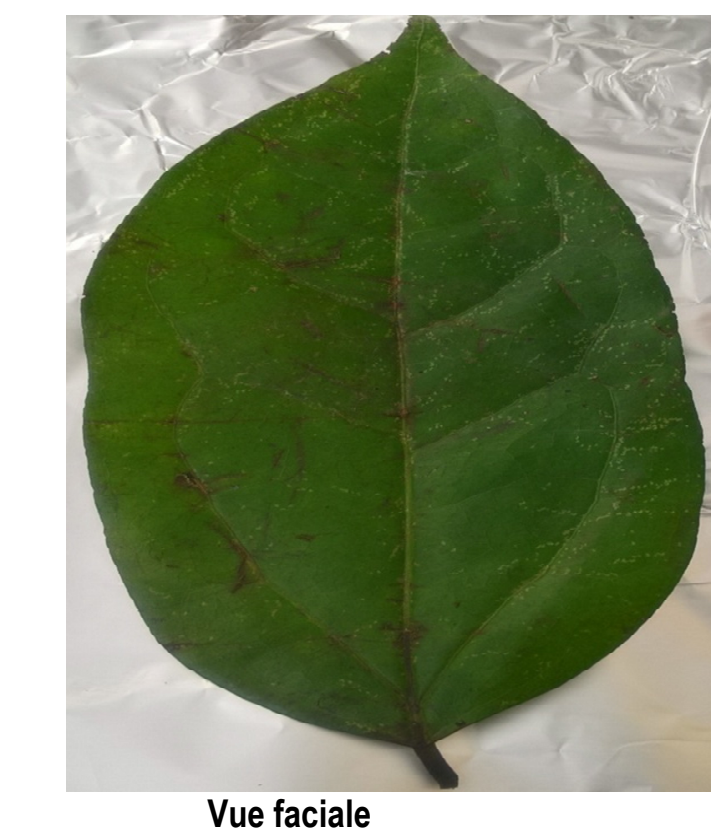

Photo 1 : Feuille de Tiliacora funifera

Le travail sur le terrain a été réalisé à travers une enquête de consommation alimentaire auprès de la de leur saveur un peu succulent. Elle utilise ces feuilles en remplacement des cubes modernes vendus dans les différents marchés dans le monde. En effet, ces feuilles rendent tendre et appétissant le saka-saka. Lors de la préparation ces feuilles sont débarrassées de la nervure principale, puis sont placées dans le mortier pour être pilées, jusqu'à avoir la forme écrasée pour certains. Soit, les feuilles entières sont placées sur le saka-saka déjà moulu avant de mettre celui-ci au feu. Les feuilles de Tiliacora funifera sont connues sous le nom de Sèkê en Téké, Musuêlè en tsangui, Niama tsaaka en Bamba, Niama tsaah en Ndassa et Lissièyi en autochtone. Cette étude est une contribution au développement des ressources naturelles encore sous -exploitées. L'objectif de cette étude consiste à caractériser les feuilles de Tiliacora funifera par la consommation et sa valeur nutritionnelle.

les renseignements sur la consommation de la plante. Le matériel biologique est constitué des feuilles de Tiliacora funifera.

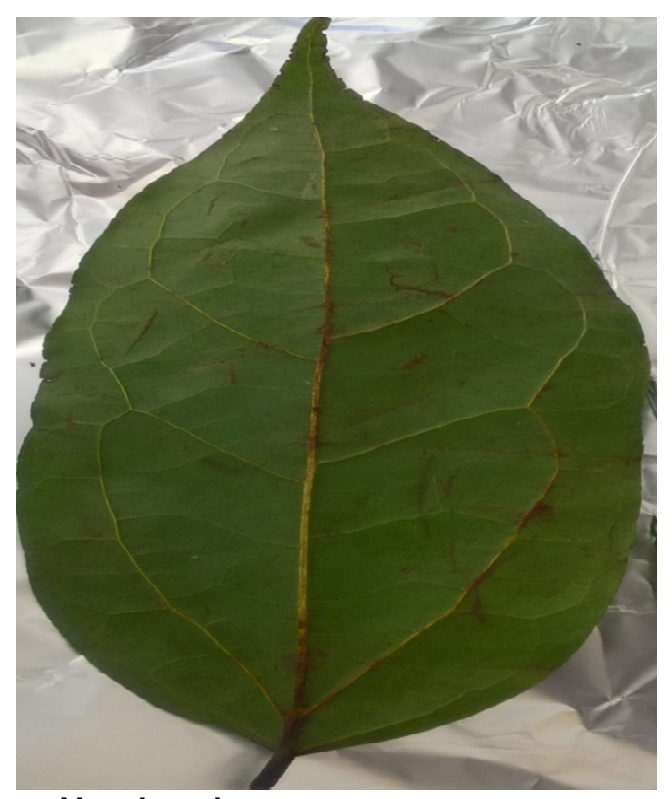

Vue dorsale

population de Komono. Elle s'est faite au moyen d'une fiche d'enquête présentée sous forme de questionnaire 
posé à l'enquêté. Les réponses ont été notées ou cochées sur la fiche par l'enquêteur. Cette fiche est composée des questions ouvertes et des questions fermées. Les grandes lignes de cette fiche sont: la connaissance de feuilles de Tiliacora funifera, la fréquence de consommation, la préférence de consommation, son utilité, les modes de préparations et leur caractères organoleptiques. A l'issue de cette enquête, les feuilles de Tiliacora funifera ont été récoltées dans la forêt primaire et/ou secondaire, protégées et emballées dans les feuilles de maranthacaes. Elles ont été transportées sous forme emballée au laboratoire pour les analyses physicochimiques.

Milieu d'étude: La zone d'étude a concerné tout Komono centre. Pour permettre d'atteindre l'ensemble de Komono centre, celui-ci a été divisée en 6 sections correspondants aux quartiers de Komono qui euxmêmes ont été subdivisés en zone de dénombrement (ZD). Chaque zone de dénombrement est constituée des plusieurs blocs, qui eux-mêmes sont constitués de plusieurs parcelles.

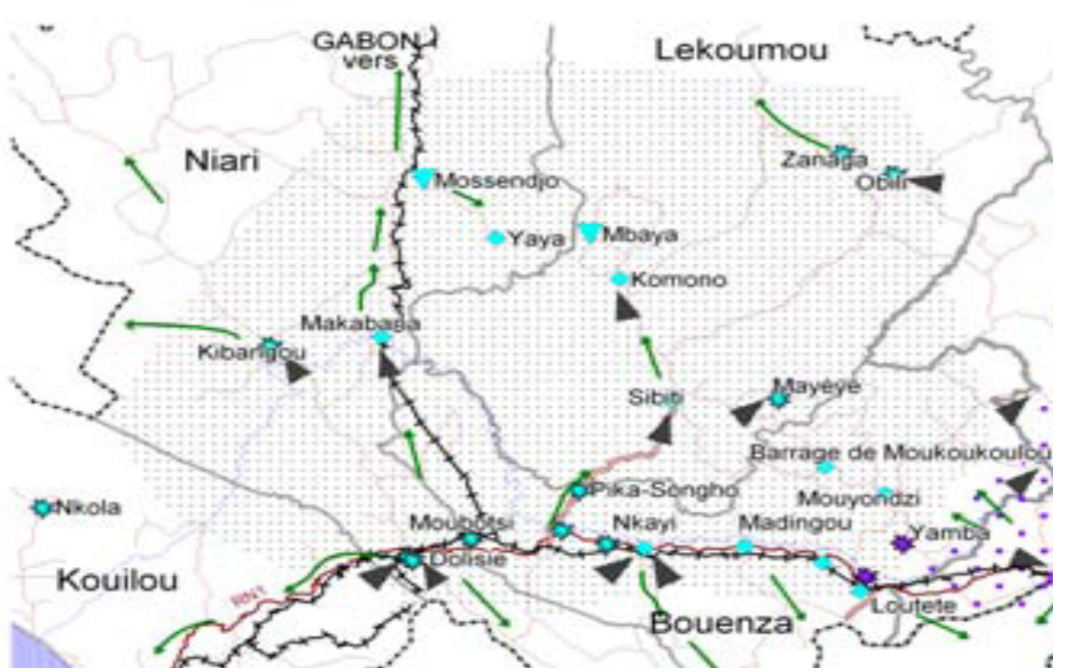

Figure 1 : Carte montrant la zone géographique de Komono (Mathilde Joncheray, Hubert Mazurek., 2011)

Domaine couvert par l'enquête : II s'agit de l'enquête de consommation des feuilles de Tiliacora funifera dans la localité de Komono centre.

Population cible : La population cible est celle vivant à Komono centre.

Base de sondage : La base de sondage est constituée de la carte de Komono. L'unité statistique est le ménage.Le ménage considéré comme unité statistique, a permis de recueillir des informations désirées (information sur les consommateurs et sur la plante) en interrogeant uniquement la personne qui consomme habituellement les feuilles de Tiliacora funifera. Seuls, les consommateurs âgés de 15 ans et plus ont été interrogés. Des quartiers ont été ensuite définis en fonction de la grandeur et de l'habitation (peuplement) du quartier pour le bloc de l'enquête. L'enquête était beaucoup plus basée dans les quartiers les plus vastes, habités et plus peuplés (les quartiers Gnimi, Centre-ville 1 et 2 (Moussangi), Mukuolo et Mouala) ensuite dans les quartiers les moins vaste comme Makélé et Lissiengué. Cette localité est constituée des différentes Ethnies: Téké, Mbamba, Ndassa, et les
Autochtones. Toutes les zones de dénombrement choisies ont été identifiées et les zones de dénombrement de travail ont été sélectionnées par tirage aléatoire.

Type d'enquête et échantillonnage : II s'est agit d'une enquête transversale. La population soumise à l'enquête a été sélectionnée sur la base d'un sondage aléatoire.

Taille de l'échantillon : La taille de l'échantillon a été de 170 ménages.

Déroulement de l'enquête : Cette enquête a consisté à rencontrer les enquêtés chez eux. Dans chaque ménage un seul individu qui s'occupe habituellement des repas a été interrogé sur la connaissance, la préparation et la consommation de ces feuilles de $T$. funifera. L'enquêté a été soumis aux questions qui suivent la chronologie de la fiche d'enquête et les réponses ont été cochées par l'enquêteur. Le dialogue s'est déroulé en langue officielle (Français) ou en langue nationale (kituba) ou encore en langue vernaculaire (téké, Bamba, Ndassa, et en autochtone) suivant la langue maîtrisée par l'enquêté. 
Traitement et Analyse des données : Le traitement des données collectées et la production des tableaux bruts ont été effectués à l'aide des logiciels Epi-Info 6fr Word, et Excel. La différence significative perçue entre deux pourcentages est vérifiée à travers les tests classiques de la statistique différentielle. La comparaison de plus de deux pourcentages a été effectuée à l'aide du test de Student (1995), représenté par la valeur de comparaison $X^{2}$; avec un seuil de significativité fixé à $5 \%$. Le travail de laboratoire a consisté à une identification de la plante au Laboratoire de l'Herbier situé à l'ORSTOM (Brazzaville). Ensuite au laboratoire pour les analyses physicochimiques sur des échantillons des feuilles de Tiliacora funifera.

Analyses physico-chimiques : Elles ont été réalisées au Laboratoire de Nutrition et Alimentation de la FST. La multiplicité a été réalisée à raison de deux essais pour chaque opération.

Détermination de la teneur en eau : La teneur en eau a été obtenue par séchage à l'étuve des feuilles de Tiliacora funifera à la température de $70^{\circ} \mathrm{C}$. $100 \mathrm{grs}$, soit la masse fraîche(Mf) des feuilles fraîche ont été pesées et placées à l'étuve pour le séchage. Le séchage a été arrêté après l'obtention de la masse sèche (Ms) constante.La teneur en eau a été obtenue par la formule suivante :

$(\% \mathrm{H})=\frac{M e}{M f} \times 100$

Avec $: \% \mathrm{H}=$ teneur en eau, $\mathrm{Me}=$ masse d'eau, $\mathrm{Mf}=$ masse fraîche

La teneur en matière sèche des feuilles de Tiliacora funifera été déterminée par la formule suivante $: \mathbf{T M}_{\mathbf{s}}(\%)$ $=\frac{M s}{M f} \times 100$

Avec: Ms = Masse sèche, $\mathrm{TMs}=$ Teneur en matière sèche

\section{Détermination de la teneur en lipide}

La teneur en lipides a été déterminée par la méthode au Soxhlet en utilisant l'hexane comme solvant d'extraction. Les feuilles de Tiliacora funifera séchées ont été broyées à l'aide d'un mortier et un pilon en porcelaine. Une masse (Mp) de 100grs du broyat (poudre) a été pesée et placée dans une cartouche. La cartouche a été introduite dans la colonne d'extraction (Soxhlet) située entre un réfrigérant et un ballon de 250 $\mathrm{ml}$ préalablement pesé (M0) et contenant $200 \mathrm{ml}$ de solvant (hexane). L'extraction de la matière grasse a été faite jusqu'à épuisement (pendant environ 5 heures). Le chauffage est arrêté après le dernier siphonage. La cartouche ne contenant plus que le tourteau est récupérée, ainsi qu'une partie du solvant (hexane) qui se trouve dans la colonne d'extraction.
L'hexane en solution avec l'huile dans le ballon a été éliminé par évaporation à l'étuve à $60^{\circ} \mathrm{C}$. Le ballon ne contenant que de l'huile a été pesé soit (M1). La masse de l'huile $(\mathrm{Mh})$ est obtenue par la relation suivante :

$\mathbf{M h}=\mathbf{M} 1-\mathbf{M} \mathbf{0}$.

La teneur en lipides se calcule selon l'équation suivante $: T_{L}(\%)=\frac{M h}{M p} \times 100$

Avec: $T_{L}=$ teneur en lipide, $M h=m a s s e$ d'huile, $M p=$ masse de la poudre

Détermination de la teneur en protéine

Dosage de l'azote : L'azote total a été déterminé par la méthode de KJELDAHL [AOAC, 1999]

Dans un matras, nous avons introduit $0,5 \mathrm{grs}$ d'échantillon végétal broyé, préalablement séché une nuit à l'étuve à $64^{\circ} \mathrm{C}$, dans lequel on ajoute une pointe de spatule de catalyseur de minéralisation et $10 \mathrm{ml}$ d'acide sulfurique concentré. Nous avons placé la solution sur la rampe de minéralisation et laisser minéraliser à froid pendant 30 minutes. Puis nous avons chauffé pendant deux heures pour une minéralisation à chaud. Nous avons laissé refroidir et avons ajouté par la suite $20 \mathrm{ml}$ d'eau distillée dans la solution et $30 \mathrm{~mL}$ d'hydroxyde de sodium à $400 \mathrm{~g} / \mathrm{l}$ jusqu'à ce que la solution devienne homogène de couleur marron. On a ensuite entraîné à la vapeur en recueillant le distillat dans un Erlen Meyer contenant 20 $\mathrm{ml}$ d'une solution d'acide borique à $20 \mathrm{~g} / \mathrm{l}$, de l'association du rouge de méthyle et le vert de Bromocrésol comme indicateurs colorés ; jusqu'à l'obtention de $125 \mathrm{ml}$. Nous avons dosé cette solution avec l'acide sulfurique à N/20 jusqu'au virage du mélange du vert au rose. Nous avons noté le volume du dosage.

\section{Calculs}

Le taux d'azote a été calculé par la formule suivante : $\% \mathrm{~N}=\frac{V H 2 S O 4 \times 0,07}{\text { Mech }}$

Avec : $\mathrm{VH}_{2} \mathrm{SO}_{4}=$ volume de l'acide sulfurique utilisé pour le dosage

Mech = masse de l'échantillon utilisé

Teneur en protéines $=\% \mathbf{N} \times 6,25$

$6,25=$ facteur de multiplication

Détermination de la teneur en cendres : Après avoir pesé la coupelle (M0) à $0,001 \mathrm{~g}$ près, $2 \mathrm{~g}$ de la poudre de Tiliacora funifera ont été pesés et placés au four à moufle et chauffé à $550^{\circ} \mathrm{C}$ pendant 8 heures. Les cendres obtenues ont été pesées. Soit Mc, la masse des cendres et $\mathrm{Mp}$, la masse de la poudre sèche. La teneur en cendres (Tc) a été déterminée par la relation suivante : 


$$
T_{C}(\%)=\frac{M c}{M p} \times 100
$$

Avec: $\mathrm{T}_{\mathrm{c}}=$ teneur en cendres, $\mathrm{Mc}_{\mathrm{c}}=$ masse des cendres, $\mathrm{Mp}=$ masse de la poudre

Détermination de la teneur en glucides totaux : La teneur en glucide a été estimée par la différence des teneurs des constituants énergétiques (lipides, protéines et cendres) avec 100\% de matières sèches, selon la formule suivante :

\section{RESULTATS}

Résultats d'enquête

Connaissance, consommation et partie consommée de la plante Tiliacora funifera: Le tableau 1
$\%$ Glucides $=100 \%-(\%$ lipides $+\%$ protéines+ $\%$ cendres)

Détermination de la valeur énergétique: Le coefficient spécifique de Merrill et Watt (1955) pour les protéines, les lipides, et les glucides a été utilisé pour la détermination de la valeur énergétique correspondante.

Tableau 1 : répartition des enquêtés selon la connaissance, consommation des feuilles de Tiliacora funifera

\begin{tabular}{l|c|c|c}
\hline \multicolumn{1}{c|}{ Paramètres } & variables & Effectif & Pourcentage (\%) \\
\hline $\begin{array}{l}\text { Connaissance des feuilles de } \\
\text { Tiliacora funifera }\end{array}$ & Oui & 170 & 100 \\
Consommation des feuilles de & 0 & 170 & 100 \\
Tiliacora funifera & Oui & 170 & 100 \\
partie consommée & Nouilles & 170 & 100 \\
But de la consommation & Rehausser le goût & 170 & \\
\hline
\end{tabular}

Le tableau 1 montre que, la plante de Tiliacora funifera est connue et consommée par toute la population de la localité de Komono (100\%). Seules ses feuilles sont consommables dans les ménages à travers des plats préparés de saka-saka dans le but de rehausser le gout et rendre tendre leur plat. représente la répartition des enquêtés sur la connaissance, la consommation ou non et la partie comestible de la plante Tiliacora funifera.

Tableau 2 : Mode d'approvisionnement et lieu de récolte des feuilles de Tiliacora funifera

\begin{tabular}{c|c|c|c|c}
\hline Paramètres & Variables & Effectif & pourcentage (\%) & Valeurs Statistiques \\
\hline Mode d'approvisionnement & Achat & 3 & 1,8 & $\mathrm{X}^{2}=146,361$ \\
& Cueillette & 167 & 98,2 & $\mathrm{ddl}=169$ \\
& Total & 170 & 100,00 & $\mathrm{p}<0,001$ \\
Lieu d'approvisionnement & marché & 3 & 1,8 & $\mathrm{X}^{2}=37,031$ \\
& savane & 0 & 0 & $\mathrm{ddl}=169$ \\
& foret & 162 & 95,3 & $\mathrm{p}<0,001$ \\
& Les deux & 5 & 2,9 & \\
\hline
\end{tabular}

Le tableau 2 montre que, $98,2 \%$ des consommateurs se procurent des feuilles de Tiliacora funifera par la cueillette contre, seulement $1,8 \%$ de ceux qui s'en procurent par achat au marché. La plupart des consommateurs récoltent les feuilles de $T$. funifera uniquement en forets $(95,3 \%), 2,9 \%$ dans les deux types de végétation (forêt et savane).Enfin, $1,8 \%$ s'en approvisionnent par achat au marché uniquement.
Modalité et lieu de récolte des feuilles de Tiliacora funifera : Le tableau 2 renseigne sur les avis des consommateurs en fonction du mode et du lieu de récolte des feuilles de Tiliacora funifera. 
Tableau 3 : différents types de forêt et de feuilles de Tiliacora funifera propres à la récolte et à la consommation

\begin{tabular}{lcccc}
\hline \multicolumn{1}{c}{ Paramètres } & Variables & Effectif & Pourcentage(\%) & Valeurs Statistiques \\
& & & & \\
\hline \multirow{2}{*}{ Types de forêt } & Foret secondaire & 21 & 12,4 & $\mathrm{X}^{2}=45,303$ \\
& Forêt vierge & 8 & 4,7 & $\mathrm{ddl}=168$ \\
& Les deux & 138 & 81,2 & $\mathrm{p}<0,001$ \\
Genre des feuilles & Total & 167 & 100,00 & \\
propres à la & Jeunes feuilles & 37 & 21,8 & $\mathrm{X}^{2}=38,303$ \\
consommation & Vielles feuilles & 18 & 10,6 & $\mathrm{ddl}=168$ \\
& Les deux & 115 & 67,6 & $\mathrm{p}<0,001$ \\
& & & & \\
\hline
\end{tabular}

II ressort des résultats du tableau 3 que, $81,2 \%$ des enquêtés récoltent les feuilles de Tiliacora funiferadans les deux types de forêts (foret secondaire et vierge ou primaire), $12,4 \%$ dans la forêt secondaire et $4,7 \%$ dans la forêt vierge. La différence est très significative car, $p<0,001$. S'agissant du genre des feuilles propres à la consommation, les résultats révèlent que $67,6 \%$ des enquêtés utilisent les deux genres des feuilles (mélange des jeunes et vieilles feuilles). $21,8 \%$ préfèrent utiliser les jeunes feuilles. Enfin, 10,6\% préfèrent les vieilles feuilles. La différence est très significative car, la probabilité $p<0,001$.

Modalité et durée de conservation: Le tableau 4 représente les différente modalités et la durée de conservation des les feuilles Tiliacora funifera.

Tableau 4 : mode, la durée et le lieu de conservation des feuilles Tiliacora funifera

\begin{tabular}{l|c|c|c|c}
\hline \multicolumn{1}{c|}{ Paramètres } & variables & Effectif & Pourcentage(\%) & Valeurs Statistiques \\
\hline Conservation des & Oui & 159 & 93,52 & $\mathrm{X}^{2}=55,016$ \\
feuilles & Non & 11 & 6,47 & $\mathrm{ddl}=168$ \\
& & & & $\mathrm{p}=<0,001$ \\
Mode de & A l'air libre & 6 & 3,77 & $\mathrm{X}^{2}=37,588$ \\
conservation & Emballé & 138 & 86,79 & $\mathrm{ddl}=168$ \\
& Autre mode & 15 & 9,43 & $\mathrm{p}=<0,001$ \\
& 2jours & 3 & 1,9 & \\
Durée de & 3jours & 18 & 11,3 & $\mathrm{X}^{2}=78,859$ \\
conservation & 7jours & 85 & 53,5 & $\mathrm{ddl}=158$ \\
& 1mois & 25 & 15,7 & $\mathrm{p}<0,001$ \\
& Plus d'un mois & 28 & 17,6 & $\mathrm{X}^{2}=78,859$ \\
Lieu de & Endroit sec & 15 & 9,4 & $\mathrm{ddl}=158$ \\
conservation & Endroit humide & 143 & 89,9 & $\mathrm{p}<0,001$ \\
& Endroit sombre & 1 & 0,6 & \\
\hline
\end{tabular}

Les résultats du tableau 4 montre que $93,52 \%$ des enquêtés conservent les feuilles de Tiliacora funifera, contre $6,47 \%$ ne les conservent pas c'est-à-dire qu'ils les utilisent le même jour après la cueillette. En ce qui concerne le mode de conservation, $86,79 \%$ les conservent sous forme emballée dans les feuilles de Marantochloa congensis, 3,77\% les laissent à l'air libre. $9,43 \%$ ont d'autres formes (en poudre par exemple). S'agissant de la durée, $53,5 \%$ des consommateurs conservent les feuilles de Tiliacora funifera pendant 7 jours, $17,6 \%$ pendant plus d'un mois, $15,7 \%$ pendant un mois, $11,3 \%$ pendant trois(03) jours. Dans l'ensemble, la différence est très significative car, la probabilité $p<0,001$. Parlant du lieu de conservation, l'endroit humide est le plus convenable pour la conservation de ces feuilles,une fois emballées dans les feuilles de Marantochloa congensis. Ce qui correspond à $89,9 \%$. Tandis que, 
$9,4 \%$ les conservent dans unendroit sec et $0,6 \%$ dans un endroit sombre. Dans l'ensemble, la différence est très significative car la probabilité $p<0,001$.
Impact sur la mauvaise conservation des feuilles de Tiliacora funifera: Le tableau 5 présente les différentes formes de détérioration sur la mauvaise conservation des feuilles de Tiliacora funifera.

Tableau 5 : différents types d'impact sur la mauvaise conservation des feuilles de Tiliacora funifera

\begin{tabular}{l|c|l|c|c}
\hline \multicolumn{1}{c|}{ Paramètres } & Variables & Effectif & Pourcentage(\%) & Valeurs Statistiques \\
\hline Impact sur la mauvaise & Oui & 106 & 62,35 & $\mathrm{X}^{2}=36,091$ \\
conservation & Non & 64 & 37,64 & $\mathrm{ddl}=169$ \\
& Total & 170 & 100,00 & $\mathrm{p}<0,001$ \\
Types d'impact sur la & Changement de & 20 & 18,86 & $\mathrm{X}^{2}=14,273$ \\
mauvaise conservation & couleur & 7 & 6,60 & $\mathrm{ddl}=169$ \\
& Flétrissement & 20 & 18,86 & $\mathrm{p}<0,001$ \\
& Ramollissement & 59 & 55,66 & \\
& Sèchement & & & \\
& TOTAL & $\mathbf{1 0 6}$ & $\mathbf{1 0 0 , 0}$ & \\
\hline
\end{tabular}

II ressort du tableau 5 que, $62,35 \%$ des enquêtés affirment qu'il y a un impact en cas de mauvaise conservation des feuilles de Tiliacora funifera, contre $37,64 \%$ qui rassurent qu'il n'y a pas d'impact sur la conservation. Car même les feuilles de Tiliacora funifera exposées à l'air libre sont toujours utilisables. Concernant l'affirmation sur l'impact, $55,66 \%$ sont pour le sèchement, contre $18,86 \%$ sont pour le changement de couleur et le ramollissement. Enfin, 6,60\% sont pour le flétrissement. Dans l' ensemble, la différence est très significative car, la probabilité $p<0,001$.

Le gout et la forme de consommation des feuilles de Tiliacora funifera ainsi que le type de plat et aliments d'accompagnements de préférence: Le tableau 6 renseigne sur le type de goût apporté par ces feuilles, la forme de consommation ainsi que les différents types de plat et aliments d'accompagnements utilisés et préférés par les consommateurs.

Tableau 6 : différents goûts et formes de consommation des feuilles de Tiliacora funifera ainsi que les types de plats et aliments d'accompagnements de préférence

\begin{tabular}{c|c|c|c|c}
\hline Paramètres & Variables & Effectif & Pourcentage(\%) & Valeurs Statistiques \\
\hline \multirow{2}{*}{ Goût procuré } & Sucré & 164 & 96,5 & $\mathrm{X}^{2}=40,066$ \\
& Amer & 1 & 0,6 & $\mathrm{ddl}=169$ \\
& Salé & 5 & 2,9 & $\mathrm{p}<0,001$ \\
& Total & 170 & 100,00 & $\mathrm{X}^{2}=239,712$ \\
Forme de consommation & Crue & 2 & 1,2 & $\mathrm{ddl}=169$ \\
& Cuite & 168 & 98,8 & $\mathrm{p}<0,001$ \\
& Total & 170 & 100,00 & $\mathrm{X}^{2}=100,478$ \\
Type de plats propres à la & Saka-saka & 167 & 98,2 & $\mathrm{ddl}=169$ \\
consommation & Gnetum & 3 & 1,8 & $\mathrm{p}<0,001$ \\
& africanum & & & \\
& & & & $\mathrm{X}^{2}=33,021$ \\
Aliment d'accompagnement & Pain & 4 & 2,4 & $\mathrm{ddl}=169$ \\
& Manioc & 143 & 84,1 & $\mathrm{p}<0,001$ \\
& Foufou & 2 & 1,2 & \\
& Riz & 12 & 7,1 & \\
\hline
\end{tabular}


Le tableau 6 montre que la plupart des consommateurs affirment que ces feuilles apportent la saveur sucrée dans leur plat de saka-saka soit $(96,5 \%)$.Par contre, $2,9 \%$ affirment avoir apporté un goût salé et $0,6 \%$ parle d'un goût amer. S'agissant de la forme de consommation, 98,8\% affirment avoir consommé les feuilles de Tiliacora funifera sous forme cuite, contre $1,2 \%$ les consomment sous la forme crue. En ce qui concerne le type, 98,2\% n'utilisent les feuilles de Tiliacora funifera que dans le plat de saka-saka et 1,8\% dans le plat de gnetum africanum. A propos de l'aliment d'accompagnement, il a été révélé que le manioc est préféré par des consommateurs, soit $(84,1) ; 7,1 \%$ avec le riz, 3,5\% avec les tubercules, $2,4 \%$ avec le pain, $1,8 \%$ avec les autres aliments (bananes plantains). Enfin 1,2\% avec le foufou. Dans l'ensemble, la différence est très significative car, la probabilité $p<0,001$.

Fréquence hebdomadaire de consommation: La figure 2 présente la fréquence de consommation hebdomadaire des feuilles de Tiliacora funifera par les enquêtés.

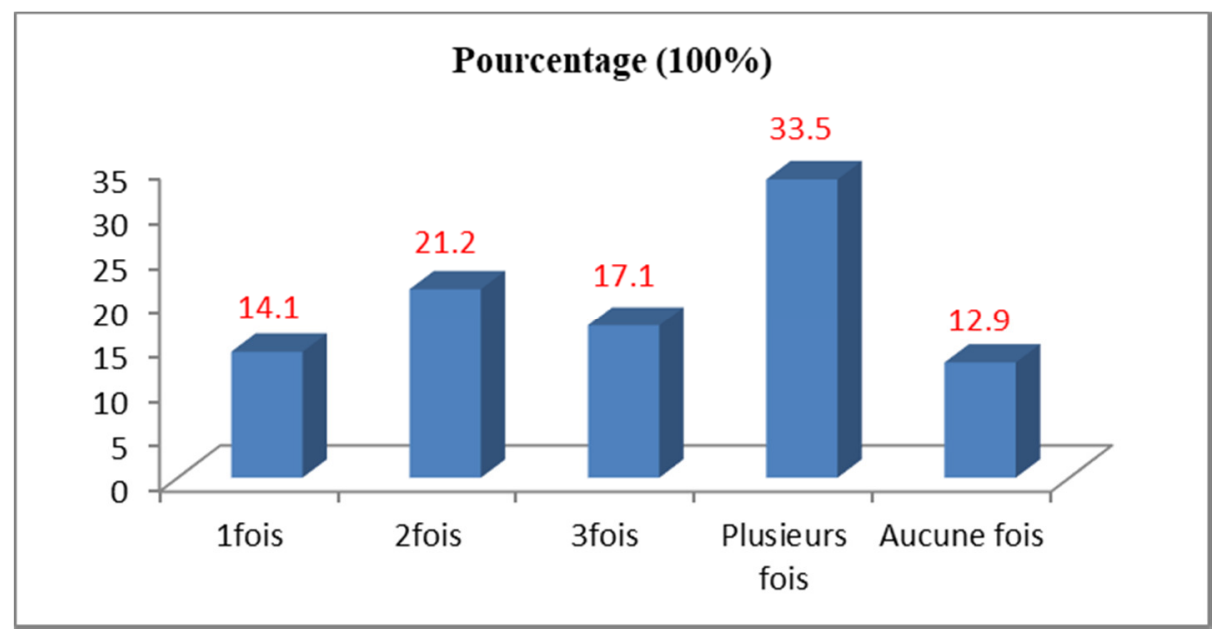

Figure 2 : répartition des enquêtés selon la fréquence hebdomadaire de consommation des feuilles de Tiliacora funifera.

Les résultats de la figure 2 montre que, 33,5\% des enquêtés consomment les feuilles de Tiliacorafunifera plusieurs fois par semaine, $21,2 \%$ les consomment 2fois par semaine, $17,1 \%$ 3fois par semaine, $14,1 \%$, 1 fois par semaine. Par contre, $12,9 \%$ des enquêtés qui n'en consomment pas pour plusieurs causes (interdiction de manger le saka-saka, l'incapacité de se déplacer, l'âge, etc). La différence est très significative car, la probabilité $p<0,001$.

Périodicité des feuilles de Tiliacora funifera : Le tableau 7 présente la saison où on trouve les feuilles en abondance dans la localité.

Tableau 7 : répartition périodicité des feuilles de Tiliacora funifera

\begin{tabular}{|c|c|c|c|c|}
\hline Paramètres & variables & Effectif & $\begin{array}{c}\text { Pourcentage } \\
(\%)\end{array}$ & $\begin{array}{c}\text { Valeurs } \\
\text { statistiques }\end{array}$ \\
\hline \multirow[t]{2}{*}{$\begin{array}{l}\text { Périodicité des } \\
\text { feuilles }\end{array}$} & $\begin{array}{l}\text { Oui } \\
\text { Non }\end{array}$ & $\begin{array}{c}11 \\
159\end{array}$ & $\begin{array}{c}6,5 \\
93,5\end{array}$ & $\begin{array}{c}x^{2}=70,975 \\
d d l=169 \\
p<0,001\end{array}$ \\
\hline & Total & 170 & 100,00 & \\
\hline Saison & $\begin{array}{l}\text { Saison sèche } \\
\text { Saison de pluies } \\
\text { TOTAL }\end{array}$ & $\begin{array}{c}2 \\
9 \\
11\end{array}$ & $\begin{array}{l}18,2 \\
81,8 \\
100\end{array}$ & $\begin{array}{c}x^{2}=14,907 \\
d d l=10 \\
p<0,001\end{array}$ \\
\hline
\end{tabular}


Les résultats du tableau 7 montrent que, 93,5\% des enquêtés ont répondus non, les feuilles de Tiliacora funifera ne sont pas saisonnières, contre $6,5 \%$ des enquêtés qui ont répondus oui. La différence est très significative car, la probabilité est $p<0,001$. Parmi les 11 enquêtés qui ont répondu oui à la périodicité de ces feuilles, $81,8 \%$ des consommateurs affirment que ces feuilles n'existent que pendant la saison des pluies, par contre, $18,2 \%$ des enquêtés affirment qu'elles ne sont présentes que pendant la saison sèche. La différence est très significative car, la probabilité est $p<0,001$.

Forme d'utilisation des feuilles de Tiliacorafunifera et différents outils: Le tableau 8 présente les différentes formes d'utilisation des feuilles Tiliacora funifera lors de la cuisson du saka-saka et les différences outils utilisés pour la forme en poudre ou morcelé.

Tableau 8 : différentes formes d'utilisation des feuilles de Tiliacora funifera et différents outils

\begin{tabular}{c|c|c|c|c}
\hline Paramètres & Variables & Effectif & $\begin{array}{c}\text { Pourcentage } \\
(\%)\end{array}$ & $\begin{array}{c}\text { Valeurs } \\
\text { statistiques }\end{array}$ \\
\hline \multirow{3}{*}{ Forme d'utilisation } & Feuilles entières & 3 & 1,8 & $\chi^{2}=76,755$ \\
& Feuilles découpées & 2 & 1,1 & $\mathrm{ddl}=169$ \\
& Feuilles broyées ou en poudre & 165 & 97,05 & $\mathrm{p}=<0,001$ \\
Modalité de pilage & Total & 170 & 100,00 & \\
\hline
\end{tabular}

II ressort de cette enquête que $97,05 \%$ des enquêtés consomment les feuilles Tiliacora funifera sous forme broyée (en poudre) en les pilant avec le mortier en bois, $3,5 \%$ mettent les feuilles morcelées et $1,8 \%$ utilisent les feuilles entières en les déposant sur le saka-saka se trouvant dans la marmite. Sur la modalité de pilage, tous les consommateurs qui broient ces feuilles, utilisent le mortier et le pilon comme outils pour écraser ces feuilles. Dans l'ensemble la différence est très significative car, la probabilité $p<0,001$.

Moment d'ajout des feuilles Tiliacora funifera dans le plat du saka-saka ainsi que les techniques de culture de la plante: Le tableau 9 présente sur le moment d'ajout des feuilles de Tilicoria funifera dans le saka-saka et les techniques de culture de la plante.

Tableau 9 : Moment d'ajout des feuilles Tiliacora funifera dans le plat du saka-saka ainsi que les techniques de culture de la plante

\begin{tabular}{c|c|c|c|c}
\hline Paramètres & variables & Effectif & Pourcentage(\%) & valeurs statistique \\
\hline $\begin{array}{c}\text { Moment d'ajout dans le } \\
\text { repas }\end{array}$ & Avant la cuisson & 163 & 95,9 & $\mathrm{X}^{2}=68,120$ \\
Culture & Pendant la cuisson & 7 & 4,1 & $\mathrm{ddl}=169$ \\
& Oui & 12 & 7,1 & $\mathrm{X}^{2}=9,001$ \\
& Non & 158 & 92,926 \\
& & & & $\mathrm{ddl}=169$ \\
& & 1 & 8,3 & $\mathrm{p}<0,001$ \\
& A travers les graines & 2 & 16,7 & $\mathrm{X}^{2}=14,182$ \\
& Par marcottage & $\mathrm{ddl}=11$ \\
& Bouturage & 9 & 75,0 & $\mathrm{p}<0,001$ \\
& Total & 12 & 100,00 & \\
\hline
\end{tabular}

Les résultats du tableau 9 montrent que, $95,9 \%$ des consommateurs ajoutent les feuilles de Tiliacora funifera avant la cuisson précisément avant de mettre la marmite au feu et $4,1 \%$ pendant la cuisson. S'agissant de la possibilité et de la technique de culture, 92,9\% confirment qu'il n' ya pas la possibilité ni technique pour cultiver la plante de Tilicoria funifera. Par contre, 7,1\% affirment que cette plante est cultivable. De la technique de culture, $8,3 \%$ ont affirmé que la culture peut se faire par graine, $16,7 \%$ ont dit qu'on peut pratiquer le marcottage. Enfin, $75 \%$ ont pensé que la multiplication peut se faire par bouturage. 
Dans l'ensemble la différence est très significative car, la probabilité $p<0,001$.

Caractéristiques morphologiques des feuilles de

Tilicoria funifera: Le tableau 10 présente les caractéristiques morphologiques des différentes variétés des feuilles de Tiliacora funifera.

Tableau 10 :Caractéristiques morphologiques des feuilles de Tilicoria funifera

\begin{tabular}{c|c|c|c|c|c}
\hline & $\begin{array}{c}\text { Longueur } \\
(\mathrm{cm})\end{array}$ & Largeur $(\mathrm{cm})$ & $\begin{array}{c}\text { Nombre de } \\
\text { nervures } \\
\text { secondaires }\end{array}$ & $\begin{array}{c}\text { Nombre de } \\
\text { nervures } \\
\text { principales }\end{array}$ & $\begin{array}{c}\text { Longueur } \\
\text { de pétiole } \\
(\mathrm{cm})\end{array}$ \\
\hline $\begin{array}{c}\text { Moyenne et } \\
\text { écart-type }\end{array}$ & $10,14 \pm 2,25$ & $6,16 \pm 2,35$ & $11 \pm 3,60$ & $1 \pm 0$ & $2,14 \pm 1,20$ \\
\hline
\end{tabular}

II ressort des résultats du tableau 10 que, les feuilles de Tiliacora funifera présentent en moyenne une longueur de $10,14 \pm 2,25 \mathrm{~cm}$, une largeur de $6,16 \pm 2,35 \mathrm{~cm}$. Le Nombre de nervures secondaires est en moyenne de $11 \pm 3,60$.
Valeur nutritive des feuilles de Tiliacora funifera : Le tableau 11 présente les qualités nutritives des feuilles de Tiliacora funifera.

Tableau 11 : Valeur nutritive des feuilles de Tiliacora funifera

\begin{tabular}{l|l|l|l|l|l|l|l}
\hline Paramètres & $\begin{array}{l}\text { Teneur en } \\
\text { eau (\%) }\end{array}$ & $\begin{array}{l}\text { Teneur } \\
\text { en MS(\%) }\end{array}$ & $\begin{array}{l}\text { Teneur en } \\
\text { protéines } \\
(\%)\end{array}$ & $\begin{array}{l}\text { Teneur en } \\
\text { glucides } \\
(\%)\end{array}$ & $\begin{array}{l}\text { Teneur en } \\
\text { lipides (\%) }\end{array}$ & $\begin{array}{l}\text { Teneur en } \\
\text { cendre(\%) }\end{array}$ & $\begin{array}{l}\text { Energie } \\
(\text { Kcal) }\end{array}$ \\
\hline $\begin{array}{l}\text { Moyenne et } \\
\text { écart type }\end{array}$ & $50,17 \pm 6,7$ & $49,81 \pm 6,7$ & 0,57 & $88,53 \pm 3,76$ & $5,50 \pm 1,5$ & $5,36 \pm 3,37$ & $406,05 \pm 10,4$ \\
\hline
\end{tabular}

II ressort du tableau 11 que, les feuilles de Tiliacora funifera ont une teneur moyenne en eau de $50,17 \pm 6,7 \%$ de matières fraiches et $49,81 \pm 6,7 \%$ de matière sèche. Par contre, les protéines et les lipides donnent des faibles teneurs moyennes respectivement

\section{DISCUSSION}

Sur la connaissance des feuilles de Tiliacora funifera, l'étude a montré que, Tiliacora funifera est une épice et condiment très bien connu par tous les enquêtés (aussi bien par les femmes, les hommes que par les différents groupes ethniques) de cette localité (100\%). Ces résultats coïncident avec les observations de Fett (2003) sur les piments (Capsicum), qui a indiqué que les piments sont les épices et les condiments les plus connus.S'agissant de la consommation, l'étude a montré que $100 \%$ des enquêtés consomment ces feuilles dans le but de rehausser le goût du plat de saka-saka en le rendant tendre et appétissant pendant la consommation. Les résultats de cette étude concordent avec ceux de Elenga et al. (2016) lors d'une étude sur Salacia pinaertii, selon lesquels, 100\% des enquêtés ont consommé ces feuilles. La feuille de Tiliacora funifera est un condiment ou épice couramment consommé à Komono dans la Lékoumou.Les différents groupes ethniques de cette de $0,57 \%$ et $5,50 \pm 1,5 \%$. Elles ont une forte teneur moyenne en glucides soit, $88,53 \pm 3,76 \%$. La teneur moyenne en cendre est de $5,36 \pm 3,37 \%$. Les feuilles de Tiliacora funifera peuvent apporter $406,05 \pm 10,4 \mathrm{Kcal}$ de l'énergie aux consommateurs.

localité consomment régulièrement ces feuilles. Ce qui est en accord avec les résultats d'Amiot-Carlin et al. (2007) qui ont relevé l'importance de l'origine ethnique (hispanique, asiatique ou afro-américaine) dans la consommation des légumes à travers une étude américaine, etceux deVan Rendsburg et al. (2005); Ambé G. A. (2001) qui stipulent qu'en Afrique, les populations font souvent recourt aux espèces végétales pour couvrir leurs besoins alimentaires et assurer leur sécurité alimentaire.Sur la partie consommée de la plante, $100 \%$ des consommateurs affirment que seules les feuilles sont consommables de toute la plante de Tiliacora funifera. Cette affirmation convient avec celle de certains auteurs comme Prosea (1993); Prota (2004) ; Kahane et al. (2005) qui stipulent que parmi les 1025 espèces cultivées ou sauvage en Afrique dont, 275 espèces proviennent de l'Afrique tropicale, 207 sont consommées pour leurs feuilles. En ce qui concerne les modalités d'approvisionnement et le lieu 
de récolte des feuilles de Tiliacora funifera, l'étude a montré que, la majorité des personnes enquêtées s'en procurent par la cueillette $(98,2 \%)$, à cause de leur disponibilité en forêt. Elles sont récoltées dans les forêts $(95,3 \%)$, précisément dans les deux types de forêt (primaire et secondaire) $(81,2 \%)$. Ces résultats se justifient par la géographie de la localité enquêtée qui est dominée par la forêt tropicale humide. Au Congo certains campagnards vivent et s'approvisionnent de produits de cueillette. Certains de ces produits sont des PFNL (comme le Gnetum africanum, les feuilles de Tiliacora funifera etc. et d'autres par contre proviennent de l'agriculture. Le Tiliacora funifera est une plante sauvage qui pousse généralement en forêt et beaucoup rencontrée dans les forêts tropicales d'Afrique centrale.A propos de la conservation, l'étude a prouvé que $93,52 \%$ des consommateurs conservent les feuilles de Tiliacora funifera emballées dans les feuilles de Marantochloa congensis(86,79\%), puis laissées dans un endroit humide $(89,9 \%)$ pendant plusieurs jours, voire des mois pour éviter tout éventuel gaspillage. Contrairement aux résultats obtenus par Hisseine (2019) lors de ces travaux sur l'évaluation nutritive des feuilles de Basella alba $L$ à Brazzaville. Ses résultats montrent que les feuilles Baselle alba $L$ sont conservées de préférence à l'air libre. En ce qui concerne la durée de conservation, les résultats de l'étude ont montré que, $53,5 \%$ des enquêtés conservent ces feuilles pendant 7 jours; $17,6 \%$ pendant plus d'un mois; $15,7 \%$ pendant un mois et $11,3 \%$ pendant trois jours. De ces faits cette durée de conservation a des impacts si celles-ci, sont mal protégées. $60,6 \%$ des consommateurs ont affirmé que la mauvaise conservation de ces feuilles, provoque les impacts suivants: le sèchement $(55,6 \%)$; le ramollissement et le flétrissement $(18,8 \%$ respectivement). $\mathrm{Au}$ regard de ces résultats, nous adhérons à l'affirmation de Kahane et al. (2005), selon laquelle« les légumes-feuilles étant périssables par leur forte teneur en eau, ils sont sensibles aux agents biologiques et physicochimique de dégradations. II s'en suit des pertes post-récoltes du point de vue quantitatif et qualitatif $» .37,6 \%$ des consommateurs affirment que, les feuilles de Tiliacora funiferane se gaspillent pas peu importe le mode conservation. D'autres formes de conservation sont la forme séchée $(67,6 \%)$ et la forme séchée réduite en poudre. Cette forme de conservation en poudre corrobore à celle de Rejeb (2008) lors de l'étude sur le curcuma.L'auteur affirme que, en cuisine, l'ensemble du rhizome est utilisé sous forme de poudre. Il est conservé dans les boites hermétiques et opaques.
Dans le même sens, Hassad (2007) affirme lors d'une étude menée sur la conservation par irradiation des épices (curcuma et poivre noir) emballées sous vide que, préserver la saveur piquante du poivre noir pendant les étapes du stockage, de la distribution et pendant sa mise sur rayon ultérieure est l' aspect le plus important auquel l'industrie doit faire face, et l'emballage doit principalement remplir cette fonction .A propos des qualités organoleptiques, les résultats ont montré que, $(96,5 \%)$ des consommateurs ont affirmé que, les feuilles de Tiliacora funifera présentent un goût sucré qui leur procure de l'appétit. Ces résultats sont supérieurs à ceux obtenus par Adialo (2017) lors d'une étude sur la caractéristique de la valeur alimentaire du Lagenaria siceraria. Ses résultats stipulent que $88,6 \%$ des consommateurs trouvent ces légumes sucré par contre $11,4 \%$ les trouvent insipides. Les feuilles de Tiliacora funifera sont parfois utilisées en remplacement du sel par certains consommateurs. Cette information rejoint celle émise par Babacar et al. (2000) sur lacomposition chimique du Nététu, condiment alimentaire produit par la fermentation des graines caroubier africain (Parkia biglobosa) Benth. L'auteur a signalé que, le nététu est parfois utilisé par les familles pauvres comme substitut de la viande.Mais trouve principalement sa place comme condiment dont les propriétés organoleptiques sont très appréciées (Ofunda, Adesomoju, 1985).S'agissant de la forme de consommation, $98,8 \%$ des consommateurs ont affirmé que, les feuilles de Tiliacora funifera sont consommées sous forme cuite, contre $1,2 \%$ sous forme crue. Nos résultats s'accordent avec ceux obtenus par Itoua etal. (2015). Ces chercheurs affirment que le Phytolacca dodecandra l'Herit est consommé sous forme cuite.A propos de l'aliment d'accompagnement, $84,1 \%$ des consommateurs consomment ces feuilles beaucoup plus avec le manioc, suivi de ceux qui en consomment aussi avec le riz $(7,1 \%)$; les tubercules $(3,5 \%)$. Cette préférence du manioc se justifie par le fait que, le manioc constitue l'aliment d'accompagnement de base dans cette localité. Nos résultats concordent avec ceux d'Itoua et al.(2015). Ces auteurs ont montré que le foufou et le manioc font partie des habitudes alimentaires des Congolais. Les feuilles de Tiliacora funifera ne sont pas consommées de façon périodique en général car la majorité $(93,5 \%)$ des enquêtés affirme que les feuilles de Tiliacora funifera sont disponibles pendant les deux saisons $(92,2 \%)$. Une légère domination de la fréquence de consommation des feuilles de Tiliacora funifera est observée pendant la saison de pluie à cause de la tombée des pluies qui 
favorisent la germination des diverses plantes et herbes. Cela montre que les feuilles condimentaires étudiées sont disponibles sur le marché quelle que soit la saison mais avec la prédominance de la saison de pluie telle que signalée par Hisseine (2019).Assobgakomlan et al.(2007) ont affirmé qu'en Afrique subsaharienne, les légumes-feuilles alimentent les marchés locaux mais aussi régionaux ». Par contre, $6,5 \%$ des enquêtés confirment la périodicité de cellesci.S'agissant du type d'assaisonnement, nos résultats montrent que $91,2 \%$ des enquêtés considèrent les feuilles de Tiliacora funifera comme condiment à cause de son apport du goût dans le saka-saka et sa disponibilité, surtout l'absence des dépenses financières. Contrairement à d'autres qui les qualifient d'épices $(7,2 \%)$ grâce à son goût, sa forme et son aspect odoriférant et gluant selon la variété. Nos résultats confirment l'observation de Fett (2003) qui a indiqué que les piments sont les épices et les condiments les plus connus. Les feuilles de Tiliacora funifera sont utilisées sous plusieurs formes dont nous avions recensé trois formes à savoir : la forme écrasée ou en poudre, cette forme est couramment utilisée par les enquêtés (femmes), elle occupe un pourcentage de 97,05 . Tous les consommateurs les pilent avec le pilon (100\%). Ce choix du pilon par rapport au moulin se justifie par l'absence du courant électrique dans la localité.

Cependant peu des enquêtés utilisent les feuilles entières et les feuilles morcelées juste à cause de la fatigue qui s'installe après leur retour des champs. Nos résultats montrent que $95,9 \%$ des enquêtés ajoutent les feuilles de Tiliacora funifera avant la cuisson du sakasaka contre $4,1 \%$ de ceux qui les ajoutent pendant la cuisson. A propos de la morphologie des feuilles, les feuilles de Tiliacora funifera présente une longueur moyenne de 10,14 cm, et une largeur de 6,16 cm. La longueur de pétiole est de $2,14 \mathrm{~cm}$, le nombre de nervures secondaires est de 11 et enfin, celui de nervures principales est de 1 . Nos travaux sont comparables à ceux obtenus par Itoua Okouango et al. (2015). Ces auteurs ont trouvé une longueur $19,92 \mathrm{~cm}$, largeur $13,87 \mathrm{~cm}$, longueur du pétiole $2,5 \mathrm{~cm}$ et le nombre de nervures 8,75 lors d'une étude sur Phytolacca dodecandra. Par contre nos travaux sont supérieurs à ceux obtenus par Loubelo (2015). L'auteur avait trouvé une longueur $7,37 \mathrm{~cm}$, largeur $5,75 \mathrm{~cm}$, longueur de pétiole $1,37 \mathrm{~cm}$ et le nombre de nervures 3,75 sur une étude de Salacia pynaertiis.S'agissant de la composition chimique, les résultats sur les analyses chimiques des feuilles de Tiliacora funifera montrent que la teneur en eau est de 50,17\%. Cette teneur est inférieure à celle obtenue par Loubelo (2015)lors d'une étude sur le Salacia pynaertii. II a obtenu $61,75 \%$ de matière fraiche. Cette différence peut s'expliquer par le temps de récolte et sur le retard pendant la période de transport de ces feuilles du lieu de récolte vers le laboratoire. Sur la teneur en matière sèche est de $49,81 \%$. Ces résultats sont largement supérieurs à ceux obtenus par Grubben (1971) pour le légume, soit une valeur de $9,9 \%$ de matière sèche. Cette différence pourrait s'expliquer par le milieu écologique et la pédologie du sol. Cette analyse est proche des résultats de la FAO (2006) qui montrent que les teneurs en matière sèche des légumes-feuilles varient entre 10 et $40 \%$ de matière sèche. Concernant la teneur en cendres, les résultats donnent $5,36 \%$. Ces résultats sont semblables à ceux obtenus par l'Association Européenne des Epices(2011) sur la table des teneurs en cendres des épices. Leurs résultats montrent la teneur en cendres de l'Allium Cepa de 50\% (oignon), $6,5 \%$ de teneur en cendre des Brassica nigra (grains de Moutarde). Cette faible teneur en cendres est observée chez la majorité des assaisonnements selon la table des teneurs en cendres présentée par l'Association Européenne des Epices. S'agissant de la teneur en protéines, les résultats de l'étude montrent que ces feuilles sont pauvres en protéines avec seulement $0,57 \%$. Ces résultats sont inférieurs à ceux obtenus par Konate M.et al. (2007) dans une étude d'évaluation des potentialités nutritives de l'Allium Cepa. L'auteur stipule que la quantité des protéines pour $100 \mathrm{~g}$ de matières sèches d'oignon est de 1,3\%. A propos de la teneur en lipides, les résultats de l'étude donnent $5,50 \%$. Cette valeur est proche de celle obtenue par Bargis et Levydutel (2015), lors d'une étude sur l'Anethum graveolens. II ressort de cette étude que cette épice (Aneth) contient une teneur en lipides de $4,4 \%$ de matières sèches. Par contre, elle est également supérieure à celle obtenue par ciqual (2013)sur l'étude de la valeur nutritionnelle pour $100 \mathrm{~g}$ de poivre noir grains et moulu. Cette étude révèle en moyenne les teneurs suivantes : $3,3 \%$ et $2,6 \%$ de matière sèche. Cette différence pourrait s'expliquer par le fait que la teneur en lipides des épices varie d'une espèce végétale à l'autre comme l'a suggéré Hisseine (2019) sur l'étude duBasella Alba $L$. Outre la variation de l'espèce végétale, la différence des teneurs en lipide pourrait s'expliquer par le fait que toutes les épices ne proviennent pas d'un même milieu écologique.Lesglucides totaux donnent une valeur de $88,53 \%$ de matières sèches. Cette valeurest supérieure 
à celle de Bargis et Levy-dutel (2015). Ces auteurs ont trouvé $68,61 \%$ de matières sèches, lors de l'étude de Piper negrum.ll est également supérieur à celui obtenu par Hassad(2007). En effet, l'auteur a trouvé une teneur en glucide de $65 \%$ matières sèches lors d'une étude sur le Curcuma Longa. Cette différence pourrait se justifier par le fait que les feuilles de Tiliacora funifera représentent un épice feuille alors que le Piper negrum est un épice en forme de graine. Cela pourrait s'expliquer aussi par l'influence des caractéristiques physico-chimiques (l'état des sols) et de la différence des plantes (facteurs génétiques).La valeur énergétique

\section{CONCLUSION ET APPLICATION DES RESULTATS}

Les feuilles de Tiliacora funifera sont beaucoup utilisées par la population de la Lékoumou dans la préparation du plat de saka-saka. Elles procurent une valeur ajoutée à ce plat en apportant une bonne saveur

\section{REFERENCES BIBLIOGRAPHIQUES}

Adialo L. S. 2017. Caractérisation de la valeur alimentaire du légume feuille Lagenaria siceraria. mémoire de fin d'étude de Master, Université Marien Ngouabi, 46p.

AmbéGuy-Alain. 2001. Les fruits sauvages comestibles des savanes guinéennes de Côte-d'Ivoire : état de la connaissance par une population locale, les Malinké.Biotechnol. Agron. Soc. Environ. 5 (1), 43-58

Amiot-Carlin MJ, Caillavet $F$, Causse M,Combris $P$, Dallongeville J, Padilla M,Renard C, Soler LG. 2007. Les fruits etlégumes dans l'alimentation. Enjeux etdéterminants de la consommation. Expertise scientifique collective, synthèsedu rapport, INRA (France). 80p.

Assogba-komlanf., Anihouvi P., Achigan E., Sikirou R., Boko A., Adje C., Ahle V., Vodouhe et Assa A. 2007.Pratique culturelle et trneur en element antinutritionnels (Nitrates et pesticide) du Salanum mocrocarpum au sud du Benin. African journal of food Agriculture Nutrition and Development, 7(4) :21p.

Association Européenne des Epices pour les plantes aromatiques et les épices. 2011. La spécification minimale des qualités.(révision $\mathrm{n}^{\circ} 4$, décembre 2011), 10p.

Babacar Ndir, Georges Lognay, Bernard Wathelet, Colette Cornelius, Michel Marlier, Philippe Thonart. 2000. Composition chimique du nététu, condiment alimentaire produit par des feuilles de Tiliacora funifera est de 406,0 Kcal. Cette valeur diffère de celle trouvée par KIMIA Food (2018). Cette société de négoce en vrac des produits et aromates, poivres et épices vendues à des industries alimentaires et pharmaceutiques, a trouvé une valeur de 508 Kcal lors de l'étude du Brassica nigra. Elle a également eu une valeur de $337 \mathrm{Kcal}$ pour l'Anisvert en poudre. Les résultats sur la composition chimiques des feuilles de Tiliacora funifera étudiées sont supérieurs à ceux de Bargis et Levy-dutel (2015), sur la composition chimique moyenne des aromates.

(bon goût). Ces feuilles auraient une bonne qualité organoleptique et méritent d'être classées dans la liste des assaisonnements (condiment et épices). Au regard de ces résultats, ces feuilles sont aussi énergétiques.

fermentation des graines du caroubier africain Parkia biglobosa (Jacq.) Benth. Biotechnologie, Agronomie, Société et Environnementvol. 4, no 2, p. 101-105.

Bailey J M. 2003. Aliments du pacifique: les feuilles vertes que nous mangeons. Version française du manuel de la CPS $n^{\circ} 31,2000$. Services de publication du secrétariat général de la Communauté du Pacifique (CPS), Graphoprint, Nouméa. 97p.

Bargis Patricia. \& Levy-Dutel Laurence. 2015. Epices, aromates, condiments et herbes aromatiques: Nutrition-Santé-Bien-être. Editions Eyrolles.

Bikoue M. Carolle, Essomba Hermann, Tabuna H., Degrande A., Tchoundjeu Z., \& Walter, S. 2007. Gestion des ressources naturelles fournissant les produits forestiers non ligneux alimentaires en Afrique Centrale (Vol. 5). Programme des Produits Forestiers non Ligneux.

Ciqual, 2013.Table de composition nutritionnelle des aliments.

Elenga M., Itoua Okouango Y. S., Loubelo Ongnangué L.U. and Mananga V. 2016.Evaluation of the consumption and physicochemical characteristics of the leaves of Salacia pynaertii in the food practices of populations of Brazzaville (Congo). African Journal of Food Science, 10(10) : 194-202.

FAO. 2006. Proximate Composition of foods. 36p. 
Fett D. D.2003. Botanical briefs :Capsicum pepper. Cutis, 72(1) : 21-3.

Gast M., Alimentation des populations de l'Ahaggar. Étude ethnographique, Paris, A.M.G., 1968. p152-169.

Grubben G.J.H. 1971. Expérimentations pour le développement de l'horticulture au SudDahomey .Rapport Tech., 3eme phase, projet FAO/CMCF Dah. 4, partie horticole, Min.Dev.Rural, Dahomey.

Hassad Héla. 2007. Conservation par irradiation des épices (poivre noir et curcuma), emballées sous vide. $117 \mathrm{p}$.

Hisseine. 2019. Caractérisation de la valeur alimentaire du légume feuille Basella Alba L. Mémoire de fin d'étude de Master, Université Marien Ngouabi, 51p.

Itoua Okouango Y.S., Elenga M., Moutsamboté J.M., Mananga V., Mbemba F. 2015.

Evaluation de la consommation et de la composition nutritionnelle des légumes feuilles de Phytolacca dodecandra L'Herit consommés par les populations originaires des districts d'Owando et de Makoua. Journal of Animal \& Plant Sciences, 27(1) : 4207-4218.

Kahane R., Temple L., Brat P., De Bon H. 2005. Les légumes-feuilles des pays tropicaux : diversité, richesse économique et valeur santé dans un contexte très fragile. Colloque Angers; les légumes : un patrimoine à transmettre et à valoriser, pp7-9.

KIMIA FOOD. 2018. Etude des épices naturelles vendues à des industries alimentaires et pharmaceutiques.

Konate M., Parkouda C., Tarpaga V., Guira F., Rouamba A., \& Sawadogo-Lingani H. 2017. Evaluation des potentialités nutritives et l'aptitude à la conservation de onze variétés d'oignon (Allium cepa L.) bulbe introduites au Burkina Faso. International Journal of
Biological and Chemical Sciences, 11(5), 2005-2015.

Latham M.C. 2001. La nutrition dans les pays en voie de développement. Rome, Italie : organisation des Nations Unis pour l'Alimentation et l'Agriculture. Collection FAO : Alimentation et Nutrition, 515p.

Loubelo Ongnangue L.U. 2015. Etude de la valeur alimentaire d'un légume de cueillette "Salacia pynaertii DE WILD»(Celestracea). Mémoire de fin d'étude de Master, Université Marien Ngouabi, 43p.

Maistre J. 1964. Les plantes à épices, Paris, G.P. Maison neuve et Larose, 290p.

Mianloundama F., Nsika Mkoko E., Loubelo E., Attibayeba, 2006. Potentiel des produits forestiers non ligneux (PFNL), Fonctionnement des filieres et contexte legale au conga brazzaville PR N³1466, 10-59.

Ofunda S., Adesomoju A. 1985. Effects of fermentation on the free fatty acids of African locust bean during iru production. J. Plant Foods 6, 111-115.

Van Rendsburg W.J, Venter S.L, Netshibuvhi T.R., Venter Heever E., Viaster H.J, de

Westphal Embrechts J. Ferwerda J. D.V., GilsMeeus H. A. E., Mustsaers H. J. W., Westphal, Stevels, J. M. C. 1985. Les cultures potagères. Culture vivrières tropicales avec références spécial au Cameroun, 321-463.

Prosea. 1993. Plant Ressources of South East Asia. Volume 8: végétales. Siemonsma J. S. et Kasem Piluek (Eds). Pudoc scientific Publishers, Wageningen, 412p.

Prota.2004. Ressources végétales de l'Afrique Tropicale, Volume 2 : légumes. Grubben G. J. H. \& Denton O. A. (Eds). Fondation PROTA/Backhuys Publishers/CTA, Wageningen, 737p.

Rejeb Imen.2008. Etude de l'effet de l'irradiation des Polyphénols du curcuma.67p. 


\section{ANNEXE : FICHE D'ENQUETE}

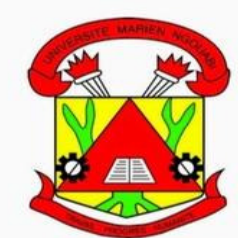

\section{UNIVERSITE MARIEN NGOUABI FACULTE DES SCIENCES ET TECHNIQUES}

Parcours : Masters sciences biologiques

Option : Nutrition Humaine

\section{Fiche d'enquête}

Titre : Enquête de consommation des feuilles de Tiliacora funifera(Sèkê) à Komono

Lieu de l'enquête

Date de l'enquête :

Quartier :

Bloc :

Zone

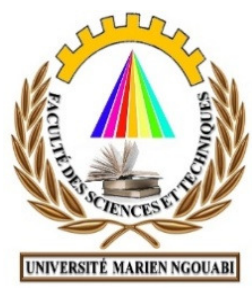

Nom de l'enquêté :

\section{Renseignement sur la plante}

1. Connaissez-vous la plante de Tiliacora funifera ? 1=oui $2=$ non

2. Consommez-vous la plante de Tiliacora funifera ? $1=$ oui $2=$ non

3. $\mathrm{Si}$ (1) quelles sont les parties consommées ? 1= feuille $2=$ tige

4. Dans quel but les consommez-vous ? 1=rehausser le gout

5. Comment le procurez-vous ?1=achat 2=récolte au maraîcher

6. Si (3) où les cueillez-vous ? 1=en forêt 2=savane

7. Si (1) quel type de foret? 1=déjà exploitée pour les travaux des champs 2=forêt vierge

8. Quels genres des feuilles cueillez-vous pour la consommation? 1=jeunes feuilles = vieilles feuilles

9. Conservez-vous ces feuilles ? $1=$ oui $2=$ non

10. Comment les conservez-vous ?1=a l'air libre 2=emballé 3=autres (précisez)

11. Combien de temps conservez-vous ces feuilles ? $1=2$ jours $\quad 2=3$ jours $3=1$ mois $4=$ plus d'un mois

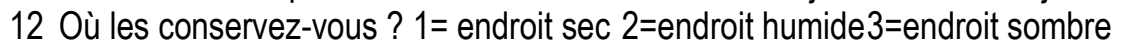

$13 \mathrm{Y}$ va-t-il un impact sur la conservation ? 1= oui $2=$ non

14 Si oui, quel impact ? 1=changement de couleur $2=$ =fétrissement $3=$ ramollissement

15 Quel est le gout de ces feuilles ? 1= sucré $\quad 2=\operatorname{amer} 3=$ salé $\quad 4=a i g r e \quad 5=$ =insipide $\quad / \quad$

16 Quelle est sa forme de consommation? 1= crue 2=cuite $3=$ =autre (précisez I I I

17 Avec quel type plat les consommez-vous ? 1=saka-saka 2=Gnetum africanum 3=autres (précisez)

1

18 Avec quel aliment de complément les consommez-vous ?1=pain $2=$ manioc $3=$ =foufou $4=l e$ riz $5=$ =tubercule 6=autres(précisez) I_ I

19 Quand avez-vous consommé ces feuilles ? $1=1$ jour $2=2$ jours $\quad 3=3$ jours $\quad 4=7$ jours $\quad 5=1$ mois $6=$ autres (précisez)

20 Combien de fois avez-vous consommé ces feuilles ? $1=1$ fois $\quad 2=2$ fois $3=3$ fois $4=$ =plusieurs fois $\quad 5=$ =aucune fois

21 Ces feuilles sont-elle saisonnières ? $\quad 1=$ oui $\quad 2=$ non

22 -Si oui, quelle saison ?1=saison sèche $2=$ saison des pluies

23 Dans quelle classe d'aliment appartiennent ces feuilles ?1=condiments 2=épices 3 3=légumes $\quad 4=a u t r e s$ (précisez)

24 Quelles autres utilisations faites-vous avec cette plante ? 1=vertus médicinal 2=autres (précisez) 3=aucune 1

25 Sous quelle forme utilisez-vous ces feuilles ? 1=feuilles entières 2=morcelées $3=$ écrasées

26 Si 1 avec quel outil le faites-vous ? 1=au couteau 2=pilon 3= au moulin 4=feuilles entières

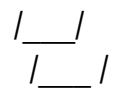


Mananga et al., J. Appl. Biosci. 2020 Évaluation de la consommation et caractérisation nutritionnelle des feuilles de Tiliacora funifera

27 A quel moment les ajoutez-vous dans le saka-saka? 1=Avant la cuisson 2=Pendant la cuisson 3=Apres la cuisson I _ I

28 Cette plante est-elle cultivable ? 1=Oui 2=Non /__ /

$29 \mathrm{Si}$ oui, comment la cultivez-vous ? 1=A travers les graines 2=par marcottage 3=par repiquage 4=parbouturage $5=$ aucune/

30 Quelle est la période à fréquence de consommation la plus élevée ? 1=saison de pluie 2=saison sèche 